\title{
WHEN IS THE UNIT TANGENT SPHERE BUNDLE SEMI-SYMMETRIC?
}

\author{
ERIC BOECKX AND GIOVANNI CALVARUSO*
}

(Received November 20, 2002)

\begin{abstract}
We prove that the unit tangent sphere bundle of a Riemannian manifold is semi-symmetric if and only if it is locally symmetric, i.e., the base manifold is either flat or it is two-dimensional with constant sectional curvature 1.
\end{abstract}

1. Introduction. The basic idea of studying geometric properties of a Riemannian manifold $(M, g)$ via those of its unit tangent sphere bundle $T_{1} M$ is well-known. In particular, many interesting interactions are known between the curvature properties of $T_{1} M$, equipped with its "natural" metric $g_{S}$ (the one induced by the Sasaki metric of the tangent bundle), and the curvature properties of $(M, g)$ itself. For explicit examples, see the survey [9] and the references therein. Moreover, $T_{1} M$ also admits a contact metric structure $(\xi, \eta, \varphi, \bar{g})$, where $\bar{g}$ is a metric homothetic to $g_{S}$. A lot of interesting results on the unit tangent sphere bundle have been obtained from the study of this structure ([1], [3], [7], [19]).

A case in point is the study of local symmetry in the context of the unit tangent sphere bundle. On the one hand, the local symmetry of the base manifold $(M, g)$ is reflected in the properties of the metric and the contact metric structure on $T_{1} M$ (see [7]), while on the other, local symmetry for $T_{1} M$ itself puts strong restrictions on the base manifold. Indeed, using contact geometry techniques, Blair [1] proved:

THEOREM 1.1. The unit tangent sphere bundle $\left(T_{1} M, \bar{g}\right)$ of a Riemannian manifold $(M, g)$ is locally symmetric if and only if either $(M, g)$ is flat or it is locally isometric to the standard sphere $S^{2}(1)$.

Note that, since $\bar{g}$ is homothetic to $g_{S},\left(T_{1} M, g_{S}\right)$ is locally symmetric if and only if $\left(T_{1} M, \bar{g}\right)$ is. An alternative proof which uses only curvature information is given in [10].

In this paper, we consider a natural generalization of the notion of local symmetry. A semi-symmetric space is a Riemannian manifold $(M, g)$ such that its curvature tensor $R$ satisfies the condition

$$
R(X, Y) \cdot R=0
$$

for all vector fields $X$ and $Y$ on $M$, where $R(X, Y)$ acts as a derivation on $R$ [21]. The name "semi-symmetric" derives from the fact that at each point $p \in M$, the curvature tensor $R_{p}$

2000 Mathematics Subject Classification. Primary 53C15; Secondary 53C25, 53C35, 53D10.

Keywords and phrases. Contact metric manifolds, semi-symmetric spaces, tangent sphere bundle, Sasaki metric.

* Supported by funds of the University of Lecce and the M.U.R.S.T. 
of $(M, g)$ is the same as that of a symmetric space (which may change with the point $p$ ). So, locally symmetric spaces are obviously semi-symmetric, but the converse is not true, as was proved by Takagi [22]. In all dimensions greater than one, there exist examples of semi-symmetric spaces which are not locally symmetric (see [6] for a survey). Nevertheless, semi-symmetry implies local symmetry in several cases. It is an interesting problem, given a class of Riemannian manifolds, to decide whether inside that class semi-symmetry implies local symmetry or not (see for example [4], [13]).

In the framework of contact Riemannian geometry, conditions of semi-symmetry have been investigated by several authors ([11], [17], [18], [23]). The second author and Perrone proved in [11] that the unit tangent sphere bundle of a Riemannian surface $M$ is semisymmetric if and only if $M$ is locally symmetric, i.e., it is either flat or it has constant curvature 1. However, their approach uses special features of the curvature of a three-dimensional contact metric manifold (see [12]) and as such cannot be simply generalized to higher dimensions. If the base manifold has dimension greater than two, a pure curvature-conditionapproach to the study of semi-symmetric unit tangent sphere bundles, starting from (1.1), seems to be extremely hard.

In this paper, we take a different road. We start from the local structure of a semisymmetric space as described by Szabó [21] and we make use of results by the first author on the local reducibility of unit tangent sphere bundles [5] to prove the following:

MAIN THEOREM. If the unit tangent sphere bundle $\left(T_{1} M, g_{S}\right)$ of a Riemannian manifold $(M, g)$ is semi-symmetric, then it is locally symmetric. Therefore, $\left(T_{1} M, g_{S}\right)$ is semisymmetric if and only if either $(M, g)$ is flat or it is locally isometric to $S^{2}(1)$.

The paper is organized in the following way. In Section 2, we recall some basic facts and results about unit tangent sphere bundles and semi-symmetric spaces. Then, we proceed to the proof of the Main Theorem. In Section 3, we consider the case of a locally irreducible unit tangent sphere bundle, while the locally reducible case is dealt with in Section 4. Finally, we generalize our result to tangent sphere bundles with arbitrary radius.

This work was initiated while the first author was visiting the University of Lecce. He wants to thank the people from the Geometry Section there for their kind hospitality during his stay.

2. Preliminaries. We restrict the information on the tangent and unit tangent sphere bundle of a Riemannian manifold to the minimum we need for this article. For a more elaborate exposition and further details, we refer to [8] and [14].

Let $(M, g)$ be a Riemannian manifold and $\pi: T M \rightarrow M$ its tangent bundle. The tangent space to $T M$ at a point $(x, u), x \in M, u \in T_{x} M$, splits into the direct sum of the vertical subspace $\operatorname{VT} M_{(x, u)}=\operatorname{ker} \pi_{* \mid(x, u)}$ and the horizontal subspace $\operatorname{HT} M_{(x, u)}$ with respect to the Levi Civita connection $\nabla$ of $M$. If $X$ is a vector field on $M$, we denote by $X^{h}$ and $X^{v}$ respectively the horizontal and the vertical lift of $X$ on $T M$. The map $X \mapsto X^{h}$ (respectively, $X \mapsto X^{v}$ ) is an isomorphism between $T_{x} M$ and $H T M_{(x, u)}$ (respectively, $T_{x} M$ 
and $\left.V T M_{(x, u)}\right)$. The Sasaki metric $g_{S}$ on $T M$ is defined by

$$
g_{S}(A, B)=g\left(\pi_{*} A, \pi_{*} B\right)+g(K A, K B),
$$

where $A, B$ are vector fields on $T M$ and $K$ is the connection map corresponding to the Levi Civita connection of $M$. Since $\pi_{*}\left(X^{h}\right)=X=K\left(X^{v}\right)$ and $\pi_{*}\left(X^{v}\right)=0=K\left(X^{h}\right)$, this is equivalent to

$$
g_{S}\left(X^{h}, Y^{h}\right)=g_{S}\left(X^{v}, Y^{v}\right)=g(X, Y), \quad g_{S}\left(X^{h}, Y^{v}\right)=0 .
$$

The tangent sphere bundle $\pi: T_{1} M \rightarrow M$ is the hypersurface of $T M$ defined by $T_{1} M=\left\{(x, u) \in T M: g_{x}(u, u)=1\right\}$. A unit normal vector field $N$ to $T_{1} M \subset T M$ is given by $N_{(x, u)}=u^{v}$. We denote again by $g_{S}$ the metric induced on $T_{1} M$ by the Sasaki metric of $T M$. We refer to [2, Chapter 9] for the description of the contact metric structure of $T_{1} M$. Here, we only recall that the contact metric of $T_{1} M$ is given by $\bar{g}=(1 / 4) g_{S}$. So, since $\bar{g}$ is homothetic to $g_{S},\left(T_{1} M, \bar{g}\right)$ is locally symmetric, respectively semi-symmetric, if and only if $\left(T_{1} M, g_{S}\right)$ has the same property.

From now on, we will always work with the Sasaki metric $g_{S}$ on $T_{1} M$. In general, the vertical lift of a vector (field) is not tangent to $T_{1} M$. For this reason, we define the tangential lift $X^{t}$ of $X \in T_{x} M$ by

$$
X_{(x, u)}^{t}=(X-g(X, u) u)^{v}=\bar{X}^{v},
$$

where we put $\bar{X}=X-g(X, u) u$ for simplicity. Clearly, tangential lifts are tangent to $T_{1} M$ and the tangent space to $T_{1} M$ is spanned by horizontal and tangential lifts of vector fields on the base manifold. The metric $g_{S}$ is then described explicitly by

$$
\begin{aligned}
& g_{S}\left(X^{t}, Y^{t}\right)=g(\bar{X}, \bar{Y})=g(X, Y)-g(X, u) g(Y, u), \\
& g_{S}\left(X^{t}, Y^{h}\right)=0, \\
& g_{S}\left(X^{h}, Y^{h}\right)=g(X, Y)
\end{aligned}
$$

at the point $(x, u) \in T_{1} M$. The Levi Civita connection $\bar{\nabla}$ associated to $g_{S}$ is given at the point $(x, u)$ by

$$
\begin{aligned}
\bar{\nabla}_{X^{t}} Y^{t} & =-g(Y, u) X^{t}, \\
\bar{\nabla}_{X^{t}} Y^{h} & =\frac{1}{2}(R(u, X) Y)^{h}, \\
\bar{\nabla}_{X^{h}} Y^{t} & =\left(\nabla_{X} Y\right)^{t}+\frac{1}{2}(R(u, Y) X)^{h}, \\
\bar{\nabla}_{X^{h}} Y^{h} & =\left(\nabla_{X} Y\right)^{h}-\frac{1}{2}(R(X, Y) u)^{t},
\end{aligned}
$$


where $R(X, Y)=\left[\nabla_{X}, \nabla_{Y}\right]-\nabla_{[X, Y]}$ is the curvature tensor of $M$. The curvature tensor $\bar{R}$ of $\left(T_{1} M, g_{S}\right)$ is given by

$$
\begin{aligned}
\bar{R}\left(X^{t}, Y^{t}\right) Z^{t}= & -g(\bar{X}, \bar{Z}) Y^{t}+g(\bar{Y}, \bar{Z}) X^{t} \\
\bar{R}\left(X^{t}, Y^{t}\right) Z^{h}= & (R(\bar{X}, \bar{Y}) Z)^{h}+\frac{1}{4}([R(u, X), R(u, Y)] Z)^{h} \\
\bar{R}\left(X^{h}, Y^{t}\right) Z^{t}= & -\frac{1}{2}(R(\bar{Y}, \bar{Z}) X)^{h}-\frac{1}{4}(R(u, Y) R(u, Z) X)^{h}, \\
\bar{R}\left(X^{h}, Y^{t}\right) Z^{h}= & \frac{1}{2}(R(X, Z) \bar{Y})^{t}-\frac{1}{4}(R(X, R(u, Y) Z) u)^{t} \\
& +\frac{1}{2}\left(\left(\nabla_{X} R\right)(u, Y) Z\right)^{h}, \\
\bar{R}\left(X^{h}, Y^{h}\right) Z^{t}= & (R(X, Y) \bar{Z})^{t} \\
& +\frac{1}{4}(R(Y, R(u, Z) X) u-R(X, R(u, Z) Y) u)^{t} \\
& +\frac{1}{2}\left(\left(\nabla_{X} R\right)(u, Z) Y-\left(\nabla_{Y} R\right)(u, Z) X\right)^{h}, \\
\bar{R}\left(X^{h}, Y^{h}\right) Z^{h}= & (R(X, Y) Z)^{h}+\frac{1}{2}(R(u, R(X, Y) u) Z)^{h} \\
& -\frac{1}{4}(R(u, R(Y, Z) u) X-R(u, R(X, Z) u) Y)^{h} \\
& +\frac{1}{2}\left(\left(\nabla_{Z} R\right)(X, Y) u\right)^{t} .
\end{aligned}
$$

Further on, we will need to know when the unit tangent sphere bundle is reducible. This question was answered recently by the first author in [5]:

THEOREM 2.1. The unit tangent sphere bundle $\left(T_{1} M, g_{S}\right)$ of a Riemannian manifold $\left(M^{n}, g\right), n \geq 2$, is locally reducible if and only if the base manifold has a flat factor, i.e., $(M, g)$ is either flat or it has a local decomposition $(M, g) \simeq\left(M^{\prime}, g^{\prime}\right) \times\left(\boldsymbol{R}^{k}, g_{0}\right)$, where $1 \leq k \leq n-2, g_{0}$ is the standard Euclidean metric on $\boldsymbol{R}^{k}$ and $\left(M^{\prime}, g^{\prime}\right)$ has no flat factor.

Next, we recall some basic facts about semi-symmetric spaces. Let $(M, g)$ be a smooth, connected Riemannian manifold. As already mentioned in the Introduction, $(M, g)$ is semisymmetric if its curvature tensor $R$ satisfies the condition (1.1). The local structure of a semisymmetric space was described by Szabó in [21]. He proves:

THEOREM 2.2. For every semi-symmetric space, there exists a dense open subset $U$ such that around every point of $U$ the manifold is locally isometric to the direct product of symmetric spaces, two-dimensional manifolds, real cones, Kählerian cones and spaces foliated by Euclidean leaves of codimension two.

Szabó arrives at this result via the study of the nullity distribution for the curvature. 
DEFINITION 2.3. The nullity vector space of the curvature tensor at a point $p$ of a Riemannian manifold $(M, g)$ is given by

$$
E_{0 p}=\left\{X \in T_{p} M \mid R(X, Y) Z=0 \text { for all } Y, Z \in T_{p} M\right\} .
$$

The index of nullity at $p$ is the number $v(p)=\operatorname{dim} E_{0 p}$. The index of non-nullity at $p$ is the number $u(p)=\operatorname{dim} M-v(p)$.

The different irreducible factors in the local decomposition theorem above correspond to different possible values for $v(p)$ and $u(p)$ :

- $\quad$ we obtain symmetric spaces for $v(p)=0$ and $u(p)>2$;

- we obtain real cones for $v(p)=1$ and $u(p)>2$;

- $\quad$ we obtain Kählerian cones for $v(p)=2$ and $u(p)>2$;

- we obtain spaces foliated by Euclidean leaves of codimension two for $v(p)=n-2$ and $u(p)=2$.

More details about these factors will be given when needed further on or they can be found in [21] and [6].

3. Semi-symmetric unit tangent sphere bundles: the irreducible case. From Theorem 1.1, we know that a Riemannian manifold $(M, g)$ which is either flat or locally isometric to the standard sphere $S^{2}(1)$ has a locally symmetric unit tangent sphere bundle $\left(T_{1} M, g_{S}\right)$. In particular, $T_{1} M$ is semi-symmetric. In the rest of this paper, we prove the converse.

The case of a two-dimensional base space $(M, g)$ was settled by the second author and Perrone in [11]. They proved:

THEOREM 3.1. Let $(M, g)$ be a two-dimensional Riemannian manifold. Its unit tangent sphere bundle $\left(T_{1} M, \bar{g}\right)$ is semi-symmetric if and only if the surface $(M, g)$ is flat or has Gaussian curvature 1.

As we already remarked, the same conclusion holds if we equip $T_{1} M$ with the Sasaki metric $g_{S}$. In the sequel, we therefore assume that the dimension of $M$ is at least three.

In this section, we suppose that $\left(T_{1} M, g_{S}\right)$ is locally irreducible. By Szabó's classification theorem above, $T_{1} M$ must be locally isometric to a symmetric space, to a real or a Kählerian cone or to a space foliated by Euclidean leaves of codimension two. We exclude these possibilities one by one.

Symmetric spaces If $\left(T_{1} M, g_{S}\right)$ is locally symmetric, the base manifold must be flat according to Theorem 1.1. But then, $\left(T_{1} M, g_{S}\right)$ is locally reducible, contrary to the assumption.

Kählerian cones Since Kählerian cones are even-dimensional (see [6]) and $T_{1} M$ is odd-dimensional, this possibility cannot occur.

Real cones We start with a more detailed description of the semi-symmetric real cones (see again [6]). These are locally isometric to the maximal Riemannian cone $M_{C}$ over a real space form $\left(M^{d}(c), g_{c}\right)$ of constant curvature $c$, for some $c \neq 1$. In particular, let $\mu(t)$ be the unique solution of the differential equation $d \mu / d t=-\mu^{2}$ with initial condition $\mu(0)=$ 
$\mu_{0}>0$, that is, $\mu(t)=\left(t+\left(1 / \mu_{0}\right)\right)^{-1}$. Put $\boldsymbol{R}_{+}=\left\{x \in \boldsymbol{R} \mid x>-1 / \mu_{0}\right\}$. On the product manifold $\boldsymbol{R}_{+} \times M^{d}(c)$, we define the Riemannian metric

$$
g_{C}=d t \otimes d t+\mu(t)^{-2} \pi_{2}^{*} g_{c},
$$

where $t$ is the natural coordinate on $\boldsymbol{R}_{+}$and $\pi_{2}: \boldsymbol{R}_{+} \times M^{d}(c) \rightarrow M^{d}(c)$ is the projection on the second factor. The resulting Riemannian space is the maximal Riemannian cone denoted by $M_{C}\left(M^{d}(c), \mu_{0}\right)$.

If we define $T:=\partial / \partial t$ to be the unit vector field tangent to $\boldsymbol{R}_{+}$on $M_{C}\left(M^{d}(c), \mu_{0}\right)$, then $T$ spans the nullity distribution $E_{0}$ of the curvature tensor $R_{C}$. For tangent vectors $X, Y$ and $Z$ orthogonal to $T$, we have

$$
R_{C}(X, Y) Z=\mu^{2}(c-1)\left(g_{C}(Y, Z) X-g_{C}(X, Z) Y\right) .
$$

Now, suppose that $\left(T_{1} M, g_{S}\right)$ is locally isometric to $M_{C}\left(M^{2 n-2}(c), \mu_{0}\right)$ for some $\mu_{0}>0$ and some $c \neq 1$. At a point $(x, u) \in T_{1} M$, denote by $T$ the unique unit vector (up to sign) in the nullity distribution $E_{0}$. We can write $T$ as $T=T_{1}^{t}+T_{2}^{h}$. Since $\operatorname{dim} M \geq 3$, we can find a non-zero tangent vector $X \in T_{x} M$ orthogonal to both $T_{1}$ and $u$. Using the curvature formulas (2.2), we have

$$
\begin{aligned}
0 & =\bar{R}\left(X^{t}, T\right) X^{t}=\bar{R}\left(X^{t}, T_{1}^{t}\right) X^{t}+\bar{R}\left(X^{t}, T_{2}^{h}\right) X^{t} \\
& =-|X|^{2} T_{1}^{t}+\bar{R}\left(X^{t}, T_{2}^{h}\right) X^{t} .
\end{aligned}
$$

Since $\bar{R}\left(X^{t}, T_{2}^{h}\right) X^{t}$ is horizontal, it follows from (3.2) that $T_{1}^{t}=0$ and $T$ must be horizontal. In particular, $T$ is orthogonal to $X^{t}$ and $Y^{t}$ for arbitrary vectors in $T_{x} M$. From (3.1) and (2.2), we find

$$
\mu^{2}(c-1)\left(|\bar{X}|^{2} Y^{t}-g(\bar{X}, \bar{Y}) X^{t}\right)=\bar{R}\left(Y^{t}, X^{t}\right) X^{t}=|\bar{X}|^{2} Y^{t}-g(\bar{X}, \bar{Y}) X^{t}
$$

and hence $\mu^{2}(c-1)=1$. As this holds at any point $(x, u) \in T_{1} M, \mu$ must be a constant function, which clearly cannot happen. Therefore, $\left(T_{1} M, g_{S}\right)$ cannot be locally isometric to a semi-symmetric real cone.

Foliated spaces Next, suppose that $\left(T_{1} M, g_{S}\right)$ is locally isometric to a space foliated by Euclidean leaves of codimension two. In particular, its index of nullity equals $2 n-3$ and its index of non-nullity equals two.

Fix a point $(x, u) \in T_{1} M$ and let $A=X_{1}^{t}+X_{2}^{h}$ be a tangent vector belonging to the nullity distribution. Take a non-zero vector $Y \in T_{x} M$ orthogonal to both $u$ and $X_{1}$. Then

$$
0=\bar{R}\left(A, Y^{t}\right) Y^{t}=|Y|^{2} X_{1}^{t}+\bar{R}\left(X_{2}^{h}, Y^{t}\right) Y^{t} .
$$

Since the second term on the right-hand side is horizontal, we conclude that $X_{1}^{t}=0$. Hence, the nullity distribution is contained in the horizontal distribution. In particular, the index of non-nullity is at least $n-1$, the dimension of the vertical distribution on $T_{1} M$. If $n>3$, this gives a contradiction.

Suppose now that $n=3$. Fix a point $x \in M$ and consider an orthonormal basis $\left\{u=e_{1}, X=e_{2}, Y=e_{3}\right\}$ of $T_{x} M$. Then, $\left\{u^{h}, X^{h}, Y^{h}, X^{t}, Y^{t}\right\}$ is an orthonormal basis 
of $T_{(x, u)} T_{1} M$. From the above, we know that the nullity distribution coincides with the horizontal distribution, i.e., $u^{h}, X^{h}, Y^{h} \in E_{0}(x, u)$. We can now show that $R=0$. Taking into account the symmetries of $R$, it is enough to show that $R_{1212}=R_{1213}=R_{1223}=R_{1313}=$ $R_{1323}=R_{2323}=0$, where $R_{i j k l}=g\left(R\left(e_{i}, e_{j}\right) e_{k}, e_{l}\right)$. Since $u^{h} \in E_{0}$, it follows from the curvature formulas (2.2) that

$$
0=4 \bar{R}\left(u^{h}, X^{t}\right) X^{t}=-\sum_{j, k=1}^{3} R_{121 j} R_{12 j k} e_{k}{ }^{h} .
$$

Hence, $R_{121 j} R_{12 j k}=0$ for all $k$. In particular, for $k=1$ we get $R_{121 j}^{2}=0$ and so, $R_{121} j=0$ for all $j$, that is, $R_{1212}=R_{1213}=0$. In the same way, we obtain $R_{1313}=0$ from $\bar{R}\left(u^{h}, Y^{t}\right) Y^{t}=0$. Next, since $X^{h}, Y^{h} \in E_{0}$, we also have

$$
\bar{R}\left(X^{h}, Y^{t}\right) Y^{t}=\bar{R}\left(Y^{h}, X^{t}\right) X^{t}=0,
$$

from which we get $R_{1323}=0$ and $R_{1223}=0$, respectively. Finally, from $\bar{R}\left(X^{h}, Y^{t}\right) X^{t}=0$, it follows that also $R_{2323}=0$. Therefore, $(M, g)$ is flat and $T_{1} M$ is locally reducible, contrary to the assumption.

4. Semi-symmetric unit tangent sphere bundles: the reducible case. In this section, we assume that the unit tangent sphere bundle $\left(T_{1} M, g_{S}\right)$ of a Riemannian manifold $\left(M^{n}, g\right), n \geq 3$, is semi-symmetric and locally reducible. According to Theorem 2.1 , there exist $k \geq 1$ and, unless $(M, g)$ is flat, a Riemannian manifold $\left(M^{\prime}, g^{\prime}\right)$ without flat factor, such that $(M, g)$ is locally isometric to the product manifold $\left(M^{\prime}, g^{\prime}\right) \times\left(\boldsymbol{R}^{k}, g_{0}\right)$. In order to prove the Main Theorem, we must show that $(M, g)$ is flat. So, we suppose it is not, i.e., $(M, g) \simeq\left(M^{\prime}, g^{\prime}\right) \times\left(\boldsymbol{R}^{k}, g_{0}\right)$ with $\operatorname{dim} M^{\prime} \geq 2$ and we derive a contradiction.

In [5], the decomposition of $\left(T_{1} M, g_{S}\right)$ is given quite explicitly. Since $M$ is a local product, a point $x$ in $M$ corresponds to a pair $\left(x^{\prime}, v_{0}\right) \in M^{\prime} \times \boldsymbol{R}^{k}$ and the tangent space $T_{x} M$ splits into the direct sum of $T_{x^{\prime}} M^{\prime}$ and $T_{v_{0}} \boldsymbol{R}^{k}$. Consider on $T_{1} M$ the following two distributions:

$$
\tilde{L}_{1}=\operatorname{ker} \pi_{*(x, u)} \oplus H_{(x, u)}\left(T_{x^{\prime}} M^{\prime}\right), \quad \tilde{L}_{2}=H_{(x, u)}\left(T_{v_{0}} \boldsymbol{R}^{k}\right) .
$$

Then, $T_{(x, u)} T_{1} M=\tilde{L}_{1} \oplus \tilde{L}_{2}$. In particular, if we denote by $X$, respectively $U$, vector fields tangent to $M^{\prime}$, respectively to $\boldsymbol{R}^{k}$, and by $A$ a generic vector field tangent to $M$, then the distribution $\tilde{L}_{1}$ is spanned by vector fields of the form $A^{t}$ and $X^{h}$, while $\tilde{L}_{2}$ is spanned by vector fields of the form $U^{h}$. From the expression (2.1) for the Levi Civita connection of $\left(T_{1} M, g_{S}\right)$, it follows easily that $\tilde{L}_{1}$ and $\tilde{L}_{2}$ are two complementary, mutually orthogonal, totally geodesic and totally parallel distributions. Therefore, the foliations $\tilde{\mathcal{L}}_{1}$ and $\tilde{\mathcal{L}}_{2}$ determined by $\tilde{L}_{1}$ and $\tilde{L}_{2}$, respectively, consist of the leaves of a local Riemannian product $M_{1} \times M_{2} \simeq T_{1} M$. Explicitly, the leaves of $\tilde{\mathcal{L}}_{1}$ are the inverse images under the natural projection $\pi$ of the leaves $\left\{M^{\prime} \times\{v\}, v \in \boldsymbol{R}^{k}\right\}$ of the product foliation on $M$, while the leaves of $\tilde{\mathcal{L}}_{2}$ are horizontal lifts of the leaves $\left\{\left\{x^{\prime}\right\} \times \boldsymbol{R}^{k}, x^{\prime} \in M^{\prime}\right\}$ of this product foliation. (Note also that the leaves of $\tilde{\mathcal{L}}_{2}$ are flat.) We refer to [5] for more details.

From these comments, it follows that we can identify $M_{1}$ with $\pi^{-1}\left(M^{\prime} \times\{0\}\right)$, and we can consider $T_{1} M^{\prime}$ as a submanifold of $M_{1}$. Clearly, the orthogonal space to $T_{1} M^{\prime}$ in $M_{1}$ is 
spanned by vertical vectors $U^{t}$, where $U$ is a vector field on $\boldsymbol{R}^{k}$. For such vector fields, we have by (2.1):

$$
\begin{aligned}
& \bar{\nabla}_{X^{t}} U^{t}=-g\left(U, u^{\prime}\right) X^{t}=0, \\
& \bar{\nabla}_{X^{h}} U^{t}=\left(\nabla_{X} U\right)^{t}+\frac{1}{2}\left(R\left(u^{\prime}, U\right) X\right)^{h}=0
\end{aligned}
$$

for all $X$ tangent to $M^{\prime}$ because of the product structure on $M$. Hence, $T_{1} M^{\prime}$ is actually a totally geodesic submanifold of $M_{1}$. Moreover, the metric induced on $T_{1} M^{\prime}$ coincides with the Sasaki metric $g_{S}^{\prime}$.

Now, if $T_{1} M \simeq M_{1} \times M_{2}$ is semi-symmetric, the same property holds for the factor $M_{1}$ and hence also for $T_{1} M^{\prime}$ as totally geodesic submanifold of a semi-symmetric space. Note that $T_{1} M^{\prime}$ cannot be reducible since by assumption $\left(M^{\prime}, g^{\prime}\right)$ has no flat factor. So, $\left(T_{1} M^{\prime}, g_{S}^{\prime}\right)$ is locally irreducible and semi-symmetric and according to the results of Section $3,\left(M^{\prime}, g^{\prime}\right)$ is locally isometric to the unit two-sphere $S^{2}(1)$. We complete the proof of the Main Theorem by proving the following:

PROPOSITION 4.1. The unit tangent sphere bundle of the Riemannian manifold $M=$ $S^{2}(1) \times \boldsymbol{R}^{k}$, with $k \geq 1$, is not semi-symmetric.

PROOF. Take an arbitrary point $x=\left(x^{\prime}, v_{0}\right) \in M=S^{2}(1) \times \boldsymbol{R}^{k}$ and a unit vector $u=\cos \theta u_{1}+\sin \theta u_{2} \in T_{x} M$, where $u_{1}$ and $u_{2}$ are unit vectors tangent to $S^{2}(1)$ at $x^{\prime}$ and to $\boldsymbol{R}^{k}$ at $v_{0}$, respectively. Let $v_{1} \in T_{x^{\prime}} S^{2}(1)$ be a unit vector orthogonal to $u_{1}$. Using the special form of the curvature tensor on $M \simeq S^{2}(1) \times \boldsymbol{R}^{k}$ and the formulas (2.2) for the curvature tensor $\bar{R}$ of $T_{1} M$, a routine calculation gives

$$
\begin{aligned}
\left(\bar{R}\left(u_{1}^{h}, u_{1}^{t}\right) \cdot \bar{R}\right)\left(u_{1}^{h}, v_{1}^{t}\right) v_{1}^{t}= & \bar{R}\left(u_{1}^{h}, u_{1}^{t}\right) \bar{R}\left(u_{1}^{h}, v_{1}^{t}\right) v_{1}^{t}-\bar{R}\left(\bar{R}\left(u_{1}^{h}, u_{1}^{t}\right) u_{1}^{h}, v_{1}^{t}\right) v_{1}^{t} \\
& -\bar{R}\left(u_{1}^{h}, \bar{R}\left(u_{1}^{h}, u_{1}^{t}\right) v_{1}^{t}\right) v_{1}^{t}-\bar{R}\left(u_{1}^{h}, v_{1}^{t}\right) \bar{R}\left(u_{1}^{h}, u_{1}^{t}\right) v_{1}^{t} \\
= & \frac{\cos ^{2} \theta}{4} \bar{R}\left(u_{1}^{h}, u_{1}^{t}\right) u_{1}^{h} \\
& -\frac{\sin ^{2} \theta}{2} \bar{R}\left(u_{1}^{h}, v_{1}^{h}\right) v_{1}^{t}-\frac{\sin ^{2} \theta}{2} \bar{R}\left(u_{1}^{h}, v_{1}^{t}\right) v_{1}^{h} \\
= & -\frac{\sin ^{2} \theta}{2} u_{1}^{t}-\frac{\sin ^{2} \theta}{4} u_{1}^{t}=-\frac{3 \sin ^{2} \theta}{4} u_{1}^{t} \\
= & -\frac{3 \sin ^{2} \theta}{4}\left(u_{1}-g\left(u, u_{1}\right) u\right)^{v} \\
= & -\frac{3 \sin ^{2} \theta}{4}\left(\sin ^{2} \theta u_{1}-\sin \theta \cos \theta u_{2}\right)^{v} .
\end{aligned}
$$

Clearly, if $\sin \theta \neq 0 \neq \cos \theta$, this is non-zero. Hence, $T_{1}\left(S^{2}(1) \times \boldsymbol{R}^{k}\right)$ is not semi-symmetric when $k \geq 1$.

5. Tangent sphere bundles with arbitrary radius. The main theorem can be easily generalized to tangent sphere bundles $T_{r} M$ with radius $r$ different from 1 . These are the 
submanifolds of $T M$ given by $T_{r} M=\left\{(x, u) \in T M: g_{x}(u, u)=r^{2}\right\}$. If we equip $T_{r} M$ with the metric induced by the Sasaki metric on $T M$ (and also denoted by $g_{S}$ ), then we obtain a Riemannian manifold which was studied, e.g., in [15] and [16]. The geometric properties of $\left(T_{r} M, g_{S}\right)$ may change with the radius. Its Levi Civita connection and Riemann curvature tensor have been calculated in [15]. One obtains expressions as (2.1) and (2.2) above, up to an occasional factor $1 / r^{2}$.

As proved in [5], Theorem 2.1 is actually valid for tangent sphere bundles $\left(T_{r} M, g_{S}\right)$ of any radius $r$. Further, it is easy to show that Theorem 1.1 by Blair has the following analogue:

THEOREM 5.1. The tangent sphere bundle $\left(T_{r} M, g_{S}\right), r>0$, of a Riemannian manifold $(M, g)$ is locally symmetric if and only if either $(M, g)$ is flat or it is locally isometric to the two-dimensional sphere $S^{2}(r)$ of radius $r$.

With these ingredients, we can now proceed as in the case of the unit tangent sphere bundle $T_{1} M$ to show:

MAIN THEOREM (general version). If the tangent sphere bundle $\left(T_{r} M, g_{S}\right), r>0$, of a Riemannian manifold $(M, g)$ is semi-symmetric, then it is locally symmetric. Therefore, $\left(T_{r} M, g_{S}\right)$ is semi-symmetric if and only if either $(M, g)$ is flat or it is locally isometric to $S^{2}(r)$.

REMARK. Of course, one can equip $T M$ with a Riemannian metric different from the Sasaki metric. One such metric appearing in the literature is the Cheeger-Gromoll metric $g_{\mathrm{CG}}$, given explicitly at the point $(x, u) \in T M$ by

$$
\begin{aligned}
& g_{\mathrm{CG}}\left(X^{h}, Y^{h}\right)=g(X, Y), \quad g_{\mathrm{CG}}\left(X^{h}, Y^{v}\right)=0, \\
& g_{\mathrm{CG}}\left(X^{v}, Y^{v}\right)=\frac{1}{1+r^{2}}(g(X, Y)+g(X, u) g(Y, u)),
\end{aligned}
$$

where $r^{2}=g_{x}(u, u)$ (see, e.g., [20]). The metric induced on the tangent sphere bundles by this metric on $T M$ does not lead to new geometric phenomena, however, since $\left(T_{r} M, g_{\mathrm{CG}}\right)$ is isometric to $\left(T_{r / \sqrt{1+r^{2}}} M, g_{S}\right)$. The isometry is given explicitly by $\phi: T_{r} M \rightarrow T_{r / \sqrt{1+r^{2}}} M$ : $(x, u) \mapsto\left(x, u / \sqrt{1+r^{2}}\right)$. In particular, it follows

THEOREM 5.2. The tangent sphere bundle $\left(T_{r} M, g_{\mathrm{CG}}\right)$ is semi-symmetric only if it is locally symmetric, i.e., if and only if the base manifold $(M, g)$ is flat or locally isometric to $S^{2}\left(r / \sqrt{1+r^{2}}\right)$.

\section{REFERENCES}

[ 1 ] D. E. BLAIR, When is the tangent sphere bundle locally symmetric?, Geometry and Topology, 15-30, World Sci. Publishing, Singapore, 1989.

[ 2 ] D. E. BLAIR, Riemannian geometry of contact and sympletic manifolds, Progr. Math. 203, Birkhäuser Boston, Inc., Boston, MA, 2002.

[ 3 ] D. E. Blair And T. Koufogiorgos, When is the tangent sphere bundle conformally flat?, J. Geom. 49 (1994), 55-66. 
[ 4 ] E. Boeckx, Einstein-like semi-symmetric spaces, Arch. Math. (Brno) 29 (1993), 235-240.

[ 5 ] E. BoEcKX, When are the tangent sphere bundles of a Riemannian manifold reducible?, Trans. Amer. Math. Soc. 355 (2003), 2885-2903.

[6] E. Boeckx, O. Kowalski And L. VAnhecke, Riemannian manifolds of conullity two, World Scientific Publishing Co., Inc., River Edge, NJ, 1996.

[ 7 ] E. Boeckx, D. Perrone And L. VAnhecke, Unit tangent sphere bundles and two-point homogeneous spaces, Period. Math. Hungar. 36 (1998), 79-95.

[ 8 ] E. Boeckx And L. VAnHecke, Characteristic reflections on unit tangent sphere bundles, Houston J. Math. 23 (1997), 427-448.

[9] E. BOECKX AND L. VANHECKE, Geometry of Riemannian manifolds and their unit tangent sphere bundles, Publ. Math. Debrecen 57 (2000), 509-533.

[10] E. BoEckX And L. VANHECKE, Unit tangent sphere bundles with constant scalar curvature, Czechoslovak Math. J. 51 (2001), 523-544.

[11] G. Calvaruso And D. Perrone, Semi-symmetric contact metric three-manifolds, Yokohama J. Math. 49 (2002), 149-161.

[12] G. Calvaruso, D. Perrone And L. Vanhecke, Homogeneity on three-dimensional contact metric manifolds, Israel J. Math. 114 (1999), 301-321.

[13] G. Calvaruso and L. Vanhecke, Semi-symmetric ball-homogeneous spaces and a volume conjecture, Bull. Austral. Math. Soc. 57 (1998), 109-115.

[14] O. KowALSKI, Curvature of the induced Riemannian metric on the tangent bundle of a Riemannian manifold, J. Reine Angew. Math. 250 (1971), 124-129.

[15] O. Kowalski And M. SekizawA, On tangent sphere bundles with small or large constant radius, Ann. Global Anal. Geom. 18 (2000), 207-219.

[16] O. Kowalski, M. Sekizawa And Z. VlášEK, Can tangent sphere bundles over Riemannian manifolds have strictly positive sectional curvature?, in: Global Differential Geometry: The Mathematical Legacy of Alfred Gray (eds. M. Fernández, J. A. Wolf), 110-118, Contemp. Math. 288, Amer. Math. Soc., Providence, RI, 2001.

[17] B. J. PApantoniou, Contact Riemannian manifolds satisfying $R(\xi, X) \cdot R=0$ and $\xi \in(k, \mu)$-nullity distribution, Yokohama Math. J. 40 (1993), 149-161.

[18] D. Perrone, Contact Riemannian manifolds satisfying $R(X, \xi) \cdot R=0$, Yokohama Math. J. 39 (1992), 141-149.

[19] D. Perrone, Tangent sphere bundles satisfying $\nabla_{\xi} \tau=0$, J. Geom. 49 (1994), 178-188.

[20] M. SEKIZAWA, Curvatures of tangent bundles with Cheeger-Gromoll metric, Tokyo J. Math. 14 (1991), $407-$ 417.

[21] Z. I. SZABÓ, Structure theorems on Riemannian manifolds satisfying $R(X, Y) \cdot R=0$, I, the local version, J. Differential Geom. 17 (1982), 531-582.

[22] H. TAKAGI, An example of Riemannian manifold satisfying $R(X, Y) \cdot R$ but not $\nabla R=0$, Tôhoku Math. J. 24 (1972), 105-108.

[23] T. TAKAHASHI, Sasakian manifolds with pseudo-Riemannian metric, Tôhoku Math. J. 21 (1969), 271-290.

[24] Y. TASHIRO, On contact structures of tangent sphere bundles, Tôhoku Math. J. 21 (1969), 117-143.

DEPARTMENT OF MATHEMATICS

KATHOLIEKE UNIVERSITEIT LEUVEN

CelestijnenlaAn 200B

3001 LEUVEN

BELGIUM

E-mail address: eric.boeckx@wis.kuleuven.ac.be
UNIVERSITÀ DEGLI STUDI DI LECCE

DipARTIMENTO Di MATEMATICA

Via PROVINCIALE LECCE-ARNESANO

73100 LECCE

ITALY

E-mail address: giovanni.calvaruso@unile.it 